\title{
Research on the Potential of Energy Internet Business Model Based on Blockchain Technology
}

\author{
Zhipeng Fan $^{1}$ \\ ${ }^{1}$ Harbin University of Commerce, Harbin, China \\ ${ }^{1}$ hsdfzp@126.com
}

\begin{abstract}
In recent years, as digital cryptocurrencies have gradually entered people's field of vision, the underlying blockchain technology has also attracted the attention of researchers. As a distributed ledger technology, blockchain has the characteristics of multi-party maintenance, non-tampering, openness and transparency. At the same time, blockchain technology is considered to be a technology with more research significance after the Internet. Blockchain technology has distributed accounting and storage, does not rely on data backup of intermediate institutions, and nodes share resources through smart contracts and are open and transparent, automatic execution, mandatory performance, and non-tamperable. This article combines blockchain and energy Internet technology. Because both have the characteristics of decentralization, high autonomy, marketization, and intelligence, they have greater application prospects. This article describes the application scenario analysis of distributed transactions in the energy Internet. First, the regional electricity sales model based on blockchain technology will be adopted to promote the implementation of distributed smart energy metering and settlement scenarios through a trustless smart contract mechanism. Secondly, it elaborates the business model of internal bidding energy consumption based on the weak centralization of blockchain technology. This model uses multi-party bidding to optimize the allocation of resources such as cold, heat, and electricity, restores the commodity and financial attributes of energy, and can greatly improve the energy efficiency and economic benefits of the microgrid.
\end{abstract}

Keywords: Blockchain; Energy Internet; Distributed; Smart Contract

\section{The development history of blockchain technology}

\subsection{Blockchain technology}

Block Chain technology is considered to be a disruptive technology with real potential for change after steam engines, electricity, and the Internet, and is expected to reshape the business form of many industries. At present, attention and research on blockchain technology from all walks of life have shown explosive growth. Blockchain technology is an integration of a series of technologies. It is a decentralized data recording and storage system formed by combining multiple technologies such as databases, digital currencies, and asymmetric encryption algorithms in a specific way. Satoshi Nakamoto proposed in 2008 that blockchain technology is a "block"-like chain data structure naturally formed in time series. Its advantage is that there is no need for a traditional central trading platform, and peer-to-peer transactions will be

Article history:

Received (August 12, 2019), Review Result (September 16, 2019), Accepted (October 20, 2019) 
realized through encryption algorithms, consensus mechanisms, time stamps and other means [1]. The blockchain network is a P2P network. There is no central hardware and management organization. The status of each node in the network is equal, and it can act as a client and a server at the same time [2]. The physical structure of a typical blockchain is shown in [Figure 1] [3].

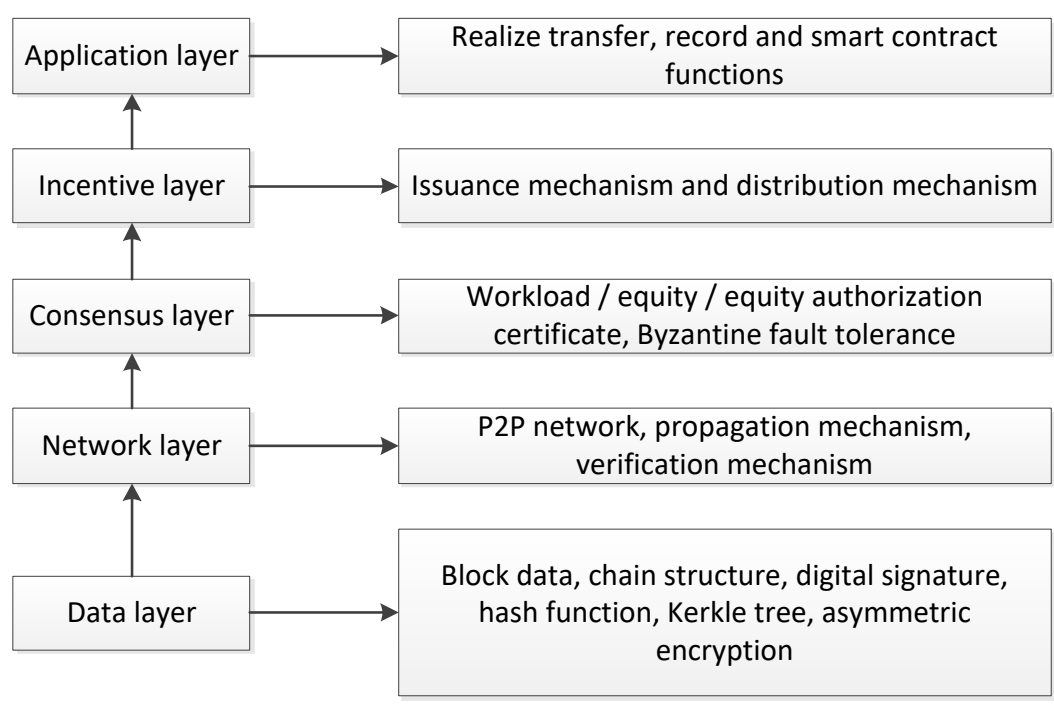

Figure 1 Blockchain technology physical structure diagram

Compared with traditional technology, blockchain technology has four major advantages: (1) Using distributed accounting and storage, system efficiency is greatly improved. (2)The nodes share resources through smart contracts, and do not rely on data backup and credit endorsements of intermediate institutions. (3) It has the characteristics of openness and transparency, automatic execution and mandatory performance. (4)It is non-tamperable, and the information is permanently sealed when added to the blockchain, and the modification of the data on a single node is invalid.

\subsection{Development history}

In 2008, "Bitcoin: A Peer-to-Peer Electronic Cash System" [4] proposed a digital currency called "Bitcoin". The original intention of Bitcoin was to make digital currency payments in an untrusted environment. Through cryptographic methods such as hash functions, asymmetric encryption, and signatures, the anonymity of users and the confirmation of transactions are realized, and the data to be maintained jointly through the consensus mechanism is reached, and a new solution to the crisis of trust is proposed. From Bitcoin Since its inception, the underlying technology of Bitcoin, the blockchain technology, has also been continuously developing. The current development of the blockchain can be divided into 3 stages.

(1) Blockchain 1.0 Blockchain 1.0 stage [5] can also be called the programmable currency stage. The blockchain enables people who do not trust each other to directly use the bits without the intervention of an authority [6] The emergence of electronic currency such as Bitcoin and the subsequent Litecoin, Dogecoin, and Ethereum has allowed the value to circulate on the Internet, and the characteristics of decentralization, cross-border payment, and anytime transactions have made digital currencies more traditional. The finance has caused a strong shock. 
(2) Blockchain 2.0 The stage of blockchain 2.0 can be called the programmable finance stage. Inspired by Bitcoin transactions, people began to try to apply blockchain to other financial fields including stocks, liquidation, private equity, etc. In April 2016, more than 80 financial institutions and supervisory members including Citibank, Deutsche Bank, HSBC, etc. formed the R3 alliance based on the blockchain platform Corda [7] released by R3. In October 2015, Nasdaq The Money20/20 conference announced the launch of the blockchain platform for private equity transactions - Linq [8], which avoids the errors that may be caused by manual liquidation and greatly reduces labor costs. In October 2015, Ripple proposed to cross Chain protocol-Inter ledger, which aims to create a unified global payment standard and simplify cross-border payment processes. The application of blockchain technology makes the financial industry hopeful to get rid of the inefficiencies caused by manual clearing, complex processes, and inconsistent standards. And high costs, disruptive changes in the traditional financial industry.

(3) Blockchain 3.0 Blockchain 3.0 stage can be called the programmable social stage. With the development of blockchain, people apply the blockchain to various fields with demand according to its characteristics. For example, application block The field of anonymous voting with the characteristics of chain anonymity, the supply chain, logistics and other fields that use the characteristics of blockchain traceability, and the Internet of Things, smart medical care, smart cities, 5G, AI and other fields. Blockchain will inevitably contribute to the future Internet And the society has a huge impact.

At present, the blockchain application technology represented by smart contracts and DAPP (distributed applications) is called the blockchain version 2.0. It pays more attention to the implementation of the real economy in application scenarios, and is an important innovation to existing business models.

\section{Blockchain Energy Internet}

Blockchain technology has enriched the original energy Internet concept and expanded it into two layers: (1) Long-distance complex and huge energy systems, such as energy transmission and sharing between global or intercontinental networks. (2) Realize the nearby transactions of regional energy. Distributed microgrid has become an important part of the large power grid, and a large number of centralized and decentralized grid topologies have been formed.

Blockchain and Energy Internet both have the characteristics of decentralization, high degree of autonomy, marketization and intelligence in technology. The $\mathrm{kWh}$ and heating and cooling energy in the blockchain mode have process records, which can realize accurate management and settlement. By sending a signal through a smart contract, energy transmission and storage will be carried out automatically, and the excess energy will be sent to the storage to ensure the real-time balance of system energy. In the future, marketization of energy commodities, diversification of supply, decentralization of systems, and smart grids will become increasingly clear development trends. Through a trustless smart contract mechanism, the blockchain can efficiently integrate various distributed energy sources and increase the penetration rate of renewable energy. It will accelerate the realization of smart grid dispatching and free market transactions, and promote the implementation of distributed smart energy metering and settlement scenarios. Therefore, blockchain technology has shown strong application potential in the field of energy Internet and new energy.

\section{Application of blockchain in the field of smart energy}




\subsection{Application scenarios}

In 2016, the "China Blockchain Technology and Application Development White Paper" issued by the Ministry of Industry and Information Technology listed 6 application scenarios with broad prospects or potential values [9]. In May 2018, Ve Chain and PricewaterhouseCoopers jointly released the "2018 China Blockchain (non-financial) Application Market Research Report", pointing out that the non-financial fields that can create the value of blockchain technology are logistics, healthcare, and energy. Etc. [10].

The smart energy system is a high degree of coupling between digital technology and the energy Internet. The core is to break all kinds of energy barriers and realize the intelligent supply of multiple energy sources. Distributed transaction is the most important application of blockchain technology in the field of smart energy, mainly in the following application scenarios:

(1) Distributed energy dispatch management

Blockchain technology based on distributed ledgers and smart contracts can realize flexible interaction between users and the power grid, and user-side loads participate in demand-side response, which will greatly enhance the flexibility and transparency of energy consumption and supply. According to reports, European transmission system operator Tennet, IBM, energy storage company Sonnen, and renewable energy company Vandebron are collaborating on a "smart grid pilot program" based on blockchain technology, and plans to use blockchain technology to manage distribution in Germany and the Netherlands. Energy to improve the balance and stability of power supply. At the EMART energy trade fair held in Amsterdam in November 2016, a total of 23 energy trading companies participated in the alliance and planned to jointly develop an energy blockchain trading system [11].

(2) Energy trading and settlement

Blockchain technology supports decentralized energy supply. Power producers, distribution operators, transmission operators and suppliers directly conduct transactions at all levels through the blockchain. Electricity settlement no longer relies on traditional power companies, individuals or companies can directly conduct transactions, and the commodity and financial attributes of energy will be further highlighted. In April 2016, the American LO3 Energy Company and Siemens Digital Grid and Bitcoin development company Consensus Systems jointly established the Brooklyn Microgrid. The micro grid is the world's first energy trading project based on blockchain technology. Users sell or purchase electricity to others through the blockchain, realizing peer-to-peer transactions that do not go through the public power company or the central grid [12].

(3) Smart power

Limited by resources and economic conditions, my country's energy consumption has long been faced with the problems of mismatch between supply and demand, shortage of flexible resources, and relatively independent energy systems. Blockchain technology is the best choice to solve these problems. The traditional energy and power industries optimize the allocation of resources based on provinces, and the provincial power dispatching and trading centers are responsible for the province's power balance. The larger the provincial energy system, the higher the cost of system decision-making [4]. Compared with the traditional energy and power industries, the energy Internet blockchain technology will develop from a centralized overall balance to a decentralized decision-making, Pareto optimal partial micro-balance. Through the digital operation of the smart grid, distributed new energy power generation can be connected to the existing power grid, and point-to-point direct transactions without centralized settlement can be realized, and resource utilization will be more intensive and efficient; it will establish a 
balance between power supply and electricity consumption. This kind of intelligent connection greatly reduces transaction costs and improves transaction efficiency [13].

(4) Energy storage electric vehicles

Energy storage Electric vehicle charging is a highly applicable sector of blockchain technology. In 2016, German power company Rheinl and Group cooperated with automotive technology company ZF and Swiss Bank to realize the use of blockchain electronic wallets to complete charging, parking and even highway toll business. This program was introduced by the Oxygen Initiative, a California start-up company in 2017, and promoted in California [14].

\subsection{Blockchain power retail will become a breakthrough}

In the future, power generation equipment will gradually show the characteristics of decentralization, and the topology of the power grid will also change with the power generation end. As a new link in the industrial chain, energy storage will play an important role in peak shaving and valley filling.

Blockchain technology can realize the digital management of energy. The production, transmission, and consumption of each kilowatt-hour of electricity have path records. The point-to-point transaction of regional power will be the most important application mode of blockchain in the energy industry. Decentralization and decentralization have realized the "direct connection" between electricity producers, electricity sales departments and consumers. Therefore, regional electricity sales based on blockchain technology will become an important breakthrough for smart energy. Following the market trend of electricity retailing, with distributed power generation flexibility trading as the entry point, electricity retailing, demandside capacity trading, operation and maintenance, and energy saving services can all be realized through off-site Internet e-commerce platforms.

\section{Smart energy business model based on blockchain}

\subsection{Smart Energy Business Model}

The characteristics of the smart energy business model based on blockchain technology are shown in [Figure 2]: (1) Realize point-to-point power transactions between users and generators. (2) Decentralized decision-making. (3) Decision-making and scheduling. The object of future power dispatch is a large number of distributed energy and smart power loads, and dispatch decisions are generated through the blockchain. (4) Realize the Pareto optimization of market competition, and the resource allocation efficiency is high [6].
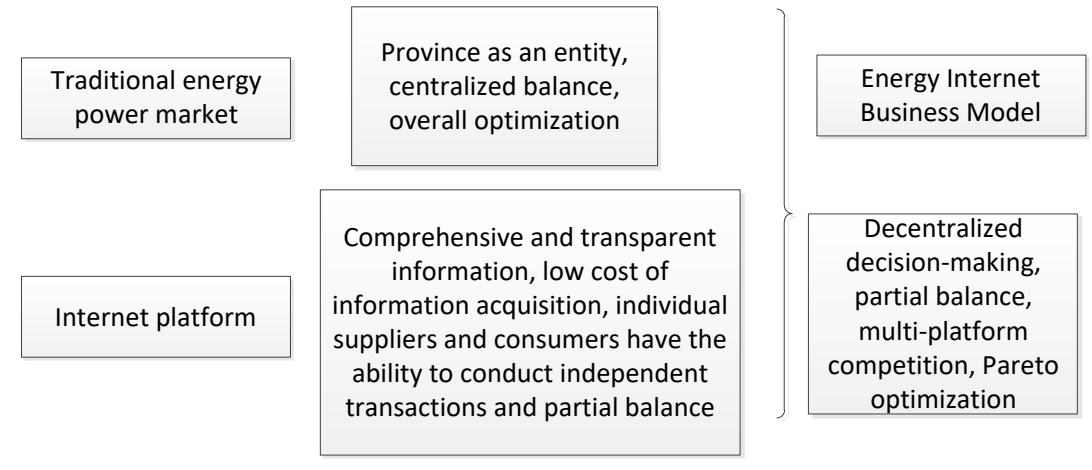

Figure 2. Characteristics of Energy Internet Business Model 


\subsection{Weakly centralized smart energy competitive energy consumption business model}

The weakly centralized smart micro-network internal bidding energy-using business model based on blockchain technology has a physical structure including energy-using layer, control layer, and functional layer. The energy consumption layer is composed of multiple industrial enterprises, household users, business districts, DC users, electric vehicles, and energy storage devices; intelligent competitive trading platform, energy Routers, two-way smart metering devices, etc. form the control layer; photovoltaic power generation, wind power/heat, gas turbine power plants, biomass power generation, ground/water source heat pumps, etc., together form the energy supply layer.

All transaction subjects have equal status in the blockchain system and are connected and interacted with each other in a flat topology. Trading energy includes cold, heat, electricity, gas, etc., and is allocated after bidding through energy routers and competitive trading platforms. [Figure 3] shows the competitive energy consumption process within the microgrid.

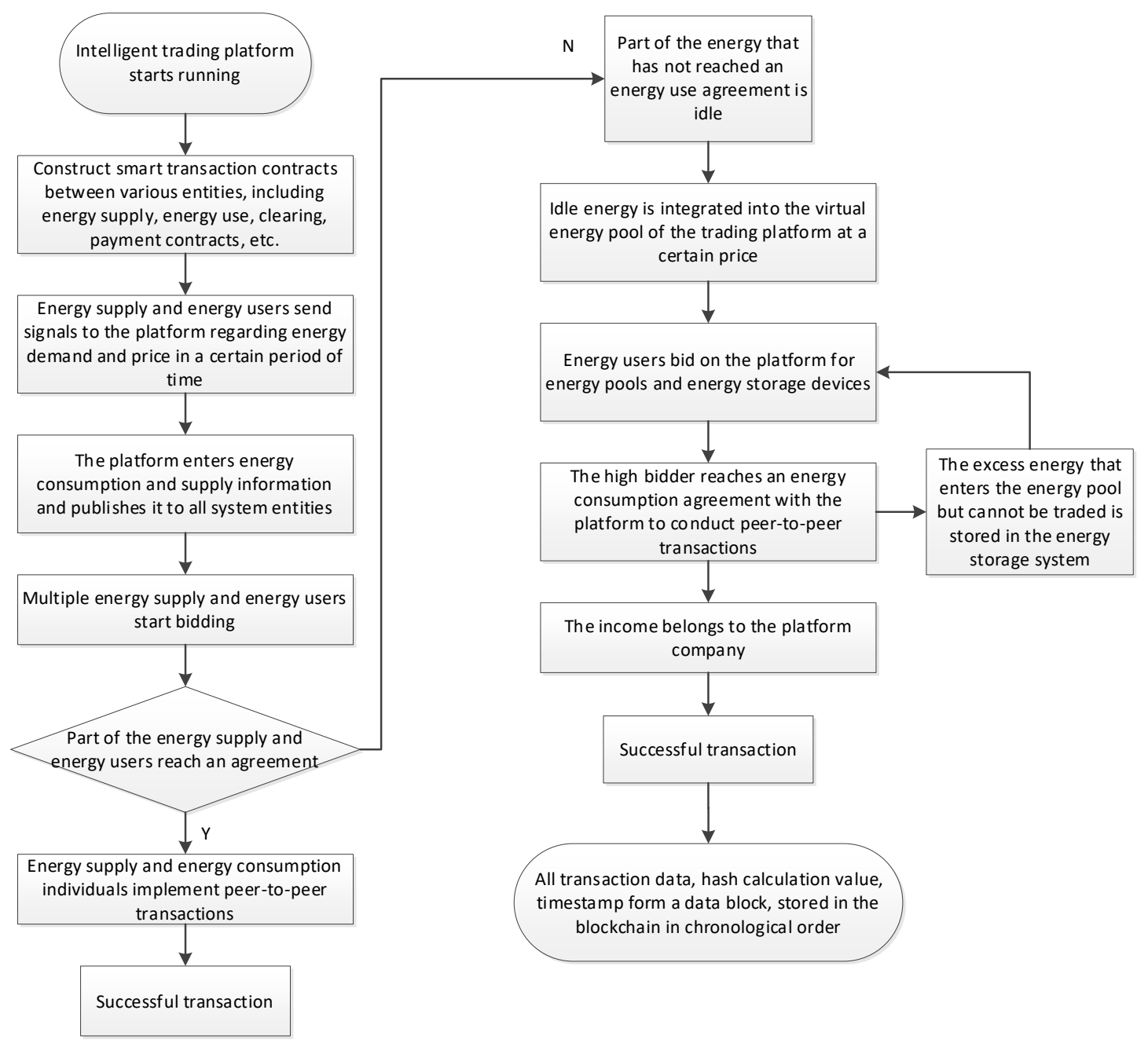

Figure 3. Blockchain-based smart energy competitive energy use flowchart 
(1) Construct smart transaction contracts between entities, including energy supply contracts, energy use contracts, storage contracts, clearing methods, payment contracts, etc.

(2) The intelligent trading platform is initialized, defining the successful transaction counter int num $1=0$, the failed transaction counter int num $2=0$, intnum $3=0$, int num $4=0$, the array numbers of both parties to the successful transaction suppy_suuccess1, consumer_success 1 , the fixed price of the energy pool double fixed_price $=0.43$ (specifically subject to the local electricity price).

(3) The trading platform receives the energy and price data sent by the energy supply and energy users and publishes them to the outside world. The energy supply array is composed of supply_id, supply_ price, and supply_output. The energy consumption array is composed of numbered consumer_id, energy pricing consumer_price, and demand consumer_consumption. The supply array of the energy supply body and the consumer array of the energy use body are assigned an initial value of -1 .

(4) In the first scenario, individual energy supply and energy users use the platform to bid on individual energy. Successful transactions need to meet the following two conditions: maximize the economic benefits of energy supply, $B_{S_{\max }}$, and minimize energy cost, $B_{C_{\min }}$. Greater than or equal to demand (supply_ output $\geq$ consumer_consumption). The bidding principles in this scenario are:

The energy supply units $S_{1}, S_{2}, S_{3} \ldots$ are $N_{s_{1}}, N_{S_{2}}, N_{S_{3}} \ldots$, and the price is $P_{S_{1}}, P_{S_{2}}, P_{S_{3}} \ldots$, The energy consumption of energy-consuming individuals $C_{1}, C_{2}, C_{3} \ldots$ is $N_{C_{1}}, N_{C_{2}}, N_{C_{3}} \ldots$, and the energy consumption price is $P_{c_{1}}, P_{c_{2}}, P_{c_{3}} \ldots$,

a) Trading principles of energy supply individuals:

$$
\left\{\begin{array}{l}
N_{S_{x}} \geq N_{C_{n}}+N_{C_{m}}+N_{C_{k}}+\cdots \\
B_{S_{\text {max }}}=\max \left(N_{C_{n}} P_{C_{n}}+N_{C_{m}} P_{C_{m}}+N_{C_{k}} P_{C_{k}}+\cdots\right)
\end{array}\right.
$$

When the energy supply meets the conditions and the $B_{S_{\max }}$ is the largest, the system will sort the energy consumption individuals $C_{n}, C_{m}, C_{k} \ldots$ according to the sort value $=$ max (energy consumption quantity $N_{C_{x}} \times$ energy consumption price $P_{C_{x}}$ ) from high to low. Subsequently, the energy supply individual $S_{x}$ sends tradable signals to the energy consuming individuals in order according to the order, and conducts transactions according to the principle of the first order transaction.

b) The trading principles of energy users:

$$
\left\{\begin{array}{l}
N_{C_{x}} \geq N_{S q}+N_{S w}+N_{S e}+\cdots \\
B_{C_{\text {min }}}=\min \left(N_{S q} P_{S q}+N_{S w} P_{S w}+N_{S e} P_{S e}+\cdots\right)
\end{array}\right.
$$

When the energy consumption meets the conditions and the $B_{C_{\min }}$ is the smallest, the system sorts the energy supply individuals $S_{q}, S_{w}, e \ldots$ according to the sort value $=$ min (energy supply quantity $N_{S x} \times$ energy supply price $P_{S x}$ ) from low to high. Subsequently, the energy consuming individuals $C_{x}$ send out tradable signals to the energy consuming individuals in order according to the ranking, and conduct transactions according to the principle of the highest priority transaction.

After the energy supply and energy use entities reach an agreement, they will conduct peerto-peer transactions. The system writes the successfully traded energy supply entities and energy use entities into the energy supply transaction array suppy_suuccess 1 and energy use transaction array consumer_success 1 .

After many cycles, the unsuccessful traded idle energy is integrated into the energy pool at a fixed price, the number of the energy supply body array is removed, and the number of the 
energy supply body that has not completed the transaction is written into the energy pool energy supply array supply_id_pool.

In this scenario, platform companies charge certain commissions to energy supply and energy consuming companies, and increase or decrease transaction commissions to encourage bidding based on the priority of the transaction.

(5) In the second scenario, the energy users and the platform bid on the energy of the platform energy pool or energy storage. The bidding principles in this scenario:

a) The energy-consuming individuals $C_{1}, C_{2}, C_{3} \ldots$ send bidding data to the platform, and their energy consumption quantities are $N_{C_{1}}, N_{C_{2}}, N_{C_{3}} \ldots$, and the energy consumption prices are $P_{c_{1}}, P_{c_{2}}, P_{c_{3}} \ldots$. The energy number provided by the platform energy pool or energy storage device is $O_{1}, O_{2}, O_{3} \ldots$, the quantity is $N_{O_{1}}, N_{O_{2}}, N_{O_{3}} \ldots$, and the price is $P_{O_{1}}, P_{O_{2}}, P_{O_{3}} \ldots$

b) The trading principles between energy users and the platform:

$$
\left\{\begin{array}{l}
N_{C_{x}} \geq N_{O d}+N_{O f}+N_{O g}+\cdots \\
B_{C_{\min }}=\min \left(N_{O d} P_{O d}+N_{O f} P_{O f}+N_{O g} P_{O g}+\cdots\right)
\end{array}\right.
$$

If the conditions are met and the $B_{C_{\min }}$ is the smallest, the system treats the individual $O_{d}, O_{f}, O_{g} \ldots$ in the platform energy pool or energy storage device. According to the sorting value $=$ min (energy supply quantity $N_{O X}$ xenergy supply price $P_{O X}$ ), they are sorted from low to high, and transactions are performed in sequence.

After a successful transaction, the remaining energy-consuming array number is the energyconsuming subject of the uncompleted transaction. Write the remaining energy consumption array consumer_id_rest, and write the energy supply number of the completed transaction into the energy pool transaction array.

When there are multiple (or single) energy suppliers and energy users, transactions are conducted on the principle of cost minimization. The calculation period $t$ for the platform to perform statistics, feedback, and judgment is tentatively set at 30s. After each individual transaction is completed, real-time judgment shall be made again, and the income from the transaction between the energy consuming individual and the platform belongs to the platform enterprise.

(6) All transaction data is packaged and stored in the data block, plus the digital fingerprint generated by the hash calculation (SHA-256) and the transaction timestamp to form a data block. All data blocks are arranged in a sequential relationship to form a chain in the system storage.

The smart energy competitive energy-using business model based on blockchain technology restores the commodity and financial attributes of energy. Establish a competitive, open, and orderly energy supply and energy market, and use multi-party bidding to optimize the allocation of resources such as cold, heat, and electricity, so as to achieve a state of competitive supply and demand balance, which can greatly improve the energy efficiency of the microgrid and benefits.

\section{Conclusion}

This article introduces the blockchain technology and its development process. On the basis of the traditional Internet, the blockchain also has characteristics that the traditional Internet does not have, such as decentralization and mutual trust. You can boldly imagine that the future Internet will present a value Internet with blockchain as the core, and blockchain will appear on top of the Internet. The software application is a new type of Internet structure on top of the blockchain. Blockchain technology, which is still in the exploratory stage, has significant 
advantages due to its decentralization, distributed decision-making, transparent performance, and intelligent settlement. The combination with "Internet + " will trigger a new round of technological revolution in the energy sector. Blockchain technology Energy Internet can solve the core problems that restrict the development of distributed energy. It is an important innovation in the business model of distributed trading. It will inject new vitality into the future development of carbon trading, microgrid, smart energy and other industries. It has played a huge role in promoting the ecology of the smart energy industry and the reconstruction of institutional mechanisms.

\section{References}

[1] NAKAMOTO S, Bitcoin: A Peer-to-peer Electronic Cash System, (2018)

[2] BAYER D, HABER S, STORNETTAW S. Improving the efficiency and reliability of digital time-stamping, Sequences II: Methods in Communication, Security and Computer Science. New York, USA: Springer-Verlag, pp.329-334, (1993)

[3] GILBERT S, LYNCH N. Brewer's conjecture and the feasibility of consistent, available, partition-tolerant Web services, ACM SIGACT News, vol.33, no.2, pp.51-59, (2002)

[4] ERKLE R C. Protocols for public key cryptosys- tems, Proceedings of the 1980IEEE Symposium on Security and Privacy(S\&P). Oakland, USA, pp.122-134, (1980)

[5] LAMPORT L. The Part-Time Parliament, ACM Transactions on Computer Systems, vol.16, no.2, pp.133-169, (1998)

[6] ONGARO D, OUSTERHOUT J. In search of an understandable consensus algorithm, The 2014 USENIX Conference on USENIX Annual Technical Conference. USENIX Association, pp.305-320, (2015)

[7] EYAL I. Blockchain Technology: Transforming Libertarian Cryptocurrency Dreams to Finance and Banking Realities, Computer, vol.50, no.9, pp.38-49, (2017)

[8] AUBLIN P L, MOKHTAR S B, QUMA V. Rbft: Redundant byzantine fault tolerance, IEEE.2013IEEE 33rd International Conference on Distributed Computing Systems. New York: IEEE, pp.297-306, (2013)

[9] EYAL I, SIRER E G. Majority is not Enough: Bitcoin Mining is Vulnerable, Communications of the ACM, vol.61, no.7, pp.95-102, (2018)

[10] REID F, HARRIGAN M. An Analysis of Anonymity in the Bitcoin System, IEEE Third International Conference on IEEE Third International Conference on Privacy.IEEE, (2012)

[11] ZYSKIND G, NATHAN O, PENTLAND A. Decentralization Privacy: Using Blockchain to Protect Personal Data, IEEE Security and Privacy Workshops, pp.180-184, (2015)

[12] MEIKLEJOHN S, POMAROLE M, JORDAN G, et al. A fistful of bitcoins: characterizing payments among men with no names, Communications of the ACM, vol.59, no.4, pp.86-93, (2016)

[13] ASSON E B, CHIESA A, GARMAN C, et al. Zerocash: Decentralized Anonymous Payments from Bitcoin, Proceedings of the 2014IEEE Symposium on Security and Privacy. NJ: IEEE, pp.459-474, (2014)

[14] KOSBA A, MILLER A, SHI E, et al. Hawk: The Blockchain Model of Cryptography and Privacy-Preserving Smart Contracts, 2016 IEEE Symposium on Security and Privacy (SP), pp.839-858, (2016) 
Research on the Potential of Energy Internet Business Model Based on Blockchain Technology

This page is empty by intention. 